\title{
Les séances d'apprentissage du raisonnement éthique appliquées à la formation des résidents en anesthésie-réanimation.
}

\author{
Ethical reasoning learning sessions applied to postgraduate anesthesiology education
}

\author{
Alexandre YAZIGI',2, Samia MADI-JEBARA', Patricia YAZBECK ${ }^{1}$
}

\begin{abstract}
Résumé Contexte : Les séances d'apprentissage au raisonnement éthique (ARE) sont une méthode de formation à l'éthique médicale. But : Décrire l'application des séances d'ARE à la formation des résidents en anesthésie-réanimation et évaluer la perception des intérêts pédagogiques de cette méthode par les participants. Sujets : Dix résidents en formation d'anesthésie ont participé aux séances d'ARE. Au cours de chaque séance, les résidents se sont réunis en groupe, en présence de deux tuteurs, pour aborder une situation clinique posant un problème éthique, vécue par un résident et sélectionnée à partir de son cahier de stage. Les discussions du groupe ont porté sur l'identification des conflits, l'inventaire des données, la délibération et la prise de décision. Un questionnaire structuré a été remis aux résidents pour évaluer leur perception des avantages pédagogiques des séances d'ARE. Résultats : Notre démarche pédagogique a inclus cinq séances d'ARE. Les ratios de réponses favorables, toujours $\geq 8 / 10$, ont montré que les séances d'ARE sont perçues comme une démarche éducative qui permet d'aborder des problèmes éthiques pertinents pour la pratique de l'anesthésie-réanimation, qui facilite l'identification des dilemmes, l'analyse des données contextuelles et la prise des décisions raisonnables et qui favorise le dialogue avec les personnes impliquées. Les résidents ont favorablement évalué le travail en petits groupes, la qualité des références et l'acquisition des concepts qui sous-tendent l'éthique médicale. Conclusion : Les séances d'ARE peuvent être appliquées à la formation des résidents en anesthésie-réanimation. Les intérêts pédagogiques de cette méthode sont favorablement perçus par les participants.
\end{abstract}

Mots clés Apprentissage au raisonnement éthique ; éthique médicale ; méthode d'apprentissage ; résidents ; anesthésie-réanimation.

Abstract Context: Ethical reasoning learning sessions are an educational method used to teach medical ethics. Goal: To describe the application of this teaching method to postgraduates education in anesthesiology and to evaluate the perceived educational relevance by participants. Subjects: Ten anesthesiology postgraduate students attended to ethical reasoning learning sessions. In each session, which was supervised by 2 tutors, participants formed an interactive group to analyze a clinical situation based on an ethical problem, which was experienced by one the participant during its training. Group discussions carried on the identification of ethical conflicts, the analysis of contextual characteristics related to the clinical case and the appropriate decision-making. At the end of each session, a structured questionnaire was distributed to participants in order to assess their perceptions regarding educational advantages. Results: Five ethical reasoning learning sessions were completed. According to collected questionnaires, more than 8/10 participants considered that this learning method allows to address relevant ethical problems related to the anesthesia practice and facilitates identification, analysis and resolution of ethical problems as well as communication among individuals who are implicated. Postgraduate students favorably perceived group discussions, the quality of the references and the acquirement of concepts related to medical ethics. Conclusion: Ethical reasoning learning sessions may be applied to anesthesiology postgraduate students education. Advantages of this method are well perceived by participants.

Key words Ethical reasoning learning sessions; teaching ethics; learning method; resident; anesthesiology.

Pédagogie Médicale 2007;8:177-83

1 Département d'anesthésie-réanimation, Hôtel-Dieu de France - Faculté de médecine, Université Saint Joseph - Beyrouth (Liban). 2 Comité d'éducation médicale, Faculté de médecine, Université Saint Joseph, Beyrouth (Liban)

Correspondance : Alexandre Yazigi, Département d’anesthésie-réanimation, Hôtel-Dieu de France, Faculté de médecine, Université Saint Joseph, Beyrouth (Liban).Téléphone :00 9611446889 - Mailto:ritalama@idm.net.lb 


\section{Concepts et Innovations}

\section{Introduction}

La capacité à identifier et prendre en compte la problématique éthique associée aux problèmes de santé est l'une des principales dimensions de la compétence professionnelle à acquérir au cours de la formation médicale $^{1,2}$. La conception de l'éthique en tant que simple application des règles déontologiques et des principes moraux a évolué vers un modèle collaboratif et dialogique $^{3,4,5}$. Dans ce modèle, l'éthique est une compétence clinique intégrée dans l'exercice quotidien de la profession médicale. Cette compétence comporte des habiletés d'identification, de délibération et de décision et elle est orientée vers le dialogue et l'action.

Dans cette perspective, les institutions académiques développent de plus en plus explicitement un enseignement structuré de l'éthique au cours de la formation médicale postdoctorale $6,7,8,9,10$. Parmi les méthodes utilisées, les séances d'apprentissage au raisonnement éthique (ARE) privilégient le travail en petits groupes et l'analyse des problèmes éthiques à partir de cas vécus et présentés par les résidents ${ }^{11}$. Le dialogue porte sur l'identification des conflits, l'inventaire des données, la délibération et la prise de décision ${ }^{12}$.

Nous avons récemment introduit les séances d'ARE dans le curriculum de nos résidents en anesthésie-réanimation, en les adaptant aux besoins spécifiques de la spécialité. Dans cet article, nous décrivons la planification et le déroulement de cette activité d'apprentissage et nous évaluons la perception de ses avantages pédagogiques par les résidents impliqués ainsi que son impact sur leur raisonnement éthique.

\section{Contexte et méthodes}

Notre faculté de médecine, dans le cadre d'une rénovation des études postdoctorales, a recommandé aux différents départements de développer des programmes de formation inspirés du concept "outcome-based education ", qui vise l'acquisition de compétences dans la collaboration, la communication, la gestion, la promotion de la santé, l'érudition et le professionnalisme. Pour répondre à cette demande institutionnelle, le département d'anesthésie-réanimation a planifié, dans une première étape, un apprentissage consacré à la formation en éthique des résidents.

La planification de cet apprentissage s'est fondée sur le cadre conceptuel pédagogique déjà adopté par notre faculté. Ce cadre inclut : 1) un apprentissage centré sur l'étudiant, qui répond à ses besoins et le motive dans sa formation ; 2) un apprentissage actif qui favorise la recherche de l'information, son élaboration et son organisation ; 3) un apprentissage contextualisé basé sur l'utilisation de problèmes réalistes et pertinents et 4) un apprentissage expérientiel développé par une démarche réflexive à partir d'expériences professionnelles vécues.

La conception de l'éthique médicale véhiculée par cet apprentissage s'est adossée sur un modèle collaboratif et dialogique, différent des approches normatives ou prescriptives. Dans ce modèle, l'éthique est ancrée dans la pratique clinique et orientée vers la réflexion, l'action et le dialogue. Elle est une composante de l'expertise professionnelle et peut être déclinée en un ensemble de compétences qui doivent résulter d'apprentissages spécifiques. Ces compétences concernent : 1) la perception et l'identification des enjeux et conflits éthiques ; 2) l'analyse et la délibération à partir des données contextuelles ; 3) la prise de décisions raisonnables, c'est-à-dire élaborée avec les personnes impliquées, cohérente avec la délibération et basée sur le discernement des circonstances réelles de la situation et 4) le maintien du dialogue avec toute entité susceptible d'être affectée par la décision. Au-delà de l'acquisition de ces compétences, cette conception de l'éthique vise la formation de futurs médecins qui combinent le "savoir " et le "vouloir " agir au bénéfice de l'autre, aussi bien singulier que collectif, dans le respect de toutes les composantes de la dignité humaine.

A partir de ces cadres conceptuels, il nous a semblé pertinent de commencer la formation en éthique par des séances d'ARE qui sont une méthode d'analyse de cas appuyée sur des situations concrètes réellement vécues et discutées en petits groupes avec des tuteurs. Les compétences en éthique, visées à l'issue de cette formation préliminaire, ne sont que partielles, encore largement en développement. Les séances d'ARE seront ultérieurement incluses dans un modèle formalisé de l'apprentissage de l'ensemble des compétences éthiques, à partir de plusieurs méthodes éducatives, tout au long du curriculum d'anesthésie-réanimation.

Les dix résidents qui étaient en formation dans notre département d'anesthésie réanimation ont participé aux séances d'ARE. Leur niveau académique variait de la première à la quatrième année postdoctorale et leurs apprentissages formels préalables en éthique médicale se limitaient à des lectures et à des discussions personnelles avec leurs superviseurs cliniques. La participation des résidents a été facultative. 
Trois enseignants du département ont contribué à la phase préparatoire des séances d'ARE. Dans une première étape, ils ont choisi quatre thèmes pertinents par rapport à la pratique de l'anesthésie-réanimation. Ces thèmes ont été : 1) le respect de l'autonomie et de la confidentialité des patients ; 2) le consentement pour un acte d'anesthésie ; 3) les enjeux de fin de vie en réanimation et 4) les questionnements liés au don d'organes. Dans une deuxième étape, ils ont revu avec les résidents, à partir de leur carnet de stage, des cas vécus et qui posaient des problèmes au niveau de l'éthique professionnelle. Dans ces cas, la responsabilité décisionnaire des résidents a été proportionnelle à leur niveau académique et a été validée par le superviseur clinique. Cinq scénarios, suffisamment élaborés, couvrant les différents thèmes d'éthique médicale déjà fixés, ont été sélectionnés. Des articles de référence en éthique, intégrant des données récentes et publiés dans des journaux d'anesthésie-réanimation, ont été proposés aux résidents. Le contenu de ces articles a inclus les concepts éthiques, les notions humanistes et les différentes approches recommandées concernant les thèmes et les cas sélectionnés.

Au cours des séances d'ARE, les résidents se sont réunis en groupe, en présence de deux tuteurs, pour aborder l'un des cas sélectionnés. Le cas a été présenté, en détail, par le résident qui l'a vécu. A partir du scénario exposé, les discussions du groupe ont porté sur les différentes étapes du raisonnement éthique : percevoir le dilemme sous-jacent et les valeurs en conflit, faire l'inventaire des données spécifiques au problème, proposer une décision raisonnable en indiquant quelle valeur privilégier et enfin établir un dialogue avec les personnes impliquées. Pour aboutir à une analyse complète des problèmes et à des consensus, les résidents ont dialogué ensemble, partagé les connaissances acquises pendant leurs lectures individuelles et mis en commun leurs expériences personnelles. Les deux tuteurs ont été des enseignants qui ont contribué à la phase préparatoire des séances d'ARE. Leur principal rôle a été de faciliter l'acquisition par les résidents des compétences éthiques visées, tout en évitant d'être eux-mêmes la principale source d'information et du dialogue. Les tuteurs ont été aidés dans leur tâche par un document contenant les concepts et les principaux éléments à aborder au cours de la discussion. A la fin de la séance, les résidents ont eu la possibilité de décrire plusieurs autres expériences professionnelles en rapport avec la problématique éthique analysée pendant la séance.

La perception, par les résidents, de l'intérêt des séances d'ARE a été évaluée par un questionnaire structuré incluant 10 items. Le questionnaire a été élaboré par les trois enseignants qui ont contribué à la phase préparatoire de la démarche. Cette élaboration s'est adossée sur les cadres conceptuels pédagogiques et éthiques adoptés lors de la planification de cet apprentissage. Les items ont porté sur : 1) les avantages pédagogiques en terme de connaissances acquises et d'apprentissage actif, motivant, contextualisé et expérientiel ; 2) les compétences visées en termes de raisonnement éthique ancré dans la pratique professionnelle et basé sur le dialogue. La compréhension des items a été testée et validée par deux résidents du département d'anesthésie. Le questionnaire a été distribué aux participants après la dernière séance d'ARE. A chacun des 10 items, ils ont répondu sur une échelle de Likert allant de 1 (= tout à fait en désaccord) à 4 (= tout à fait d'accord). Les réponses au questionnaire ont été individuelles et anonymes.

Limpact de la démarche pédagogique sur la progression du raisonnement éthique des résidents a été évalué par les deux tuteurs. Cette évaluation, de type informel et formatif, a eu lieu en groupe, à la fin de chacune des séances d'ARE. Elle a porté sur l'identification des dilemmes et des valeurs en conflits, l'inventaire des données contextuelles, la qualité de la délibération et sa cohérence avec de la décision.

\section{Résultats}

Notre démarche pédagogique s'est déroulée sur cinq semaines et a inclus cinq séances d'ARE. Le nombre de participants a été de huit à neuf résidents par séance. Les scénarios des cas abordés sont présentés dans le tableau 1. La durée d'une séance d'ARE a été de 60 à 90 minutes.

Dix questionnaires remis aux résidents ont été rendus et analysés. Les items de ce questionnaire sont présentés dans le tableau 2. Les réponses «tout à fait d'accord " ou " plutôt d'accord " ont été considérées comme des réponses favorables. Les ratios de réponses favorables ont montré que les résidents ont perçu les séances d'ARE comme une démarche éducative qui permet d'aborder des scénarios et des problèmes éthiques pertinents pour la pratique de l'anesthésie-réanimation (8/10), qui facilite l'identification des dilemmes éthiques (9/10), l'analyse des multiples données contextuelles $(9 / 10)$ et la prise de décisions raisonnables $(9 / 10)$ et qui favorise le dialogue et l'écoute des personnes impliquées $(8 / 10)$. Les résidents ont favorablement évalué le travail en petits groupes $(8 / 10)$, la qualité des références $(8 / 10)$ et l'acquisition des principes et des concepts qui sous-tendent l'éthique médicale (9/10). 


\section{Concepts et Innovations}

\section{Tableau 1 : \\ Les scénarios des cas abordés lors des cinq séances d'apprentissage du raisonnement éthique}

1- Un témoin de Jéhovah en chirurgie cardiaque

2- L'obtention du consentement pour une anesthésie locorégionale

3- L'arrêt des thérapeutiques chez un patient en réanimation

4- Le respect de la confidentialité chez un patient HIV positif

5- Le don d'organes d'un traumatisé en mort cérébrale

\section{Tableau 2 :}

\section{Les items du questionnaire d'évaluation}

Concernant les 4 séances d'apprentissage au raisonnement éthique (ARE) auxquelles vous avez participé, répondez aux questions suivantes par (1 à 4$)$ :

$1:$ Si vous êtes en désaccord

2 : Si vous êtes plutôt en désaccord

3 : Si vous êtes plutôt d'accord

4: Si vous êtes d'accord

1- Les thèmes abordés (consentement éclairé, limitation des soins, don d'organes etc.) sont en lien avec votre pratique d'anesthésie réanimation.

2- Les cas cliniques discutés sont réalistes et correspondent à des cas que vous avez vécu.

3- Les références mises à votre disposition sont adéquates.

4- Le travail en petit groupe, avec un tuteur, est une bonne méthode pour l'apprentissage de l'éthique.

5- L'interactivité en petit groupe (échange, discussion, écoute etc.) a été satisfaisante.

6- Les séances d'ARE vous ont permis de mieux comprendre les principes et les concepts qui soustendent l'éthique médicale.

7- Les séances d'ARE vous aideront à mieux percevoir l'existence d'un dilemme éthique au cours de votre pratique médicale.

8- Les séances d'ARE vous permettront de mieux analyser et pondérer les différentes données d'un problème éthique rencontré au cours de votre pratique.

9- Les séances d'ARE vous aideront à prendre la ou les décisions convenables pour résoudre un conflit éthique rencontré au cours de votre pratique médicale.

10- Les séances d'ARE vous ont sensibilisé à l'importance du dialogue avec les personnes impliquées dans un conflit éthique rencontré au cours de votre pratique. 
Les deux tuteurs qui ont évalué l'impact de la démarche pédagogique sur le raisonnement éthique des résidents au cours des séances d'ARE ont rapporté que, au terme de cette démarche : 1) l'identification des dilemmes et des valeurs en conflit est plus claire ; 2) l'inventaire des données pertinentes au cas est plus complet ; 3 ) la délibération est mieux élaborée et 4) les décisions proposées sont, au-delà de leurs contenus, plus cohérentes avec l'analyse des données contextuelles et avec la délibération au sein du groupe.

\section{Discussion}

Ce travail rapporte une démarche éducative basée sur des séances d'ARE adaptées à la formation des résidents en anesthésie-réanimation. Les cas discutés ont été sélectionnés à partir d'exemples vécus et rapportés par les apprenants. Les scénarios ont représenté des situations couramment rencontrées dans la pratique de l'anesthésie-réanimation. Les cas ont été suffisamment variés, complexes et riches en données pour couvrir les problèmes éthiques pertinents pour l'exercice de la spécialité. Les textes de références proposés aux résidents ont abordé les principes et les questionnements éthiques dans le contexte spécifique de leur spécialité. Les tuteurs du groupe ont été des anesthésistes universitaires, impliqués dans le programme de formation des résidents et ayant suivi une formation en éthique médicale.

Les méthodes d'enseignement de l'éthique au cours de la formation des résidents en anesthésie-réanimation ont été rarement rapportées dans la littérature. Parmi ces méthodes, Edelstein et al. ${ }^{10}$ ont décrit des présentations magistrales suivies de séances de discussion de cas. La Société américaine d'anesthésie, dans son syllabus sur l'éthique (http://www.asahq.org/publications AndServices/EthicsSyllabus.pdf) ${ }^{13}$, propose des sessions de discussion de cas à partir d'une série de questions préétablies. Comparées à ces méthodes de type "case-based", les séances d'ARE appliquées à la formation de nos résidents d'anesthésie nous semblent pouvoir offrir plusieurs avantages : 1) la présentation d'un cas réellement vécu situe les résidents dans leur propre pratique et favorise l'apprentissage contextualisé de l'éthique ; 2) les discussions en petits groupes encouragent le dialogue et la délibération dans l'action et à propos de l'action ; 3) l'analyse basée sur les étapes du raisonnement éthique facilite l'acquisition d'une compétence éthique intégrée dans l'exercice quotidien de la profession médicale ${ }^{12}$.

La perception de l'intérêt de notre démarche pédago- gique de la part des résidents est étayée par les résultats du questionnaire. Les séances d'ARE sont appréciées par les résidents qui estiment avoir amélioré leur capacité à développer un raisonnement éthique lors des discussions en petits groupes, jugement qui est accrédité par l'appréciation des deux tuteurs. Plusieurs auteurs ont rapporté la perception et l'impact favorable des méthodes d'apprentissage de l'éthique basée sur l'analyse de cas et sur les discussions avec les pairs. Ces résultats ont été retrouvés chez des résidents en médecine ${ }^{14}$, en chirurgie ${ }^{7}$, en obstétrique ${ }^{6}$ et en médecine de famille ${ }^{15}$. L'impact positif de ces méthodes d'enseignement sur le raisonnement éthique a aussi été décrit au cours des études prédoctorales ${ }^{16,17,18}$.

L'expérience pédagogique que nous rapportons est limitée. Elle n'a inclus que cinq séances d'ARE, dix résidents et deux tuteurs. L'évaluation que nous en rapportons est également très partielle. Nos résultats sont donc relativement préliminaires. Pour être mieux jugée, notre démarche pédagogique devrait être appliquée pendant une période plus longue et devrait porter sur un nombre beaucoup plus important de cas d'éthique et concerner davantage de résidents d'anesthésie. Les résultats de notre évaluation pédagogique auraient pu être enrichis par une approche qualitative utilisant d'autres méthodes de recueil d'informations telles qu'un questionnaire ouvert ou des entretiens semi-structurés. Le faible effectif des participants et le caractère exploratoire de l'étude constituaient à cet égard des conditions favorables. Pour autant, une telle approche n'aurait pas nécessairement permis de documenter l'impact réel de la séquence pédagogique sur la capacité des étudiants à recontextualiser les apprentissages effectués en situation authentique de pratique professionnelle. Du reste, aucun des divers instruments d'évaluation du raisonnement éthique précédemment décrits ${ }^{19,20}$, ne permet complètement une telle appréciation. Dans cette perspective, l'observation directe des résidents au cours de leur stage clinique et l'évaluation du contenu de leur portfolio sont tenues pour constituer des méthodes plus valides ${ }^{11}$. Enfin, l'activité éducative que nous rapportons ne permet pas de préciser la place des séances d'ARE par rapport aux autres moyens d'apprentissage de l'éthique médicale, tels que la supervision clinique avec rétroaction, le portfolio, les conférences et l'influence des enseignants " modèles de rôles ".

Il est logique de soutenir que des activités optimales d'enseignement et d'apprentissage de l'éthique dans un curriculum post-doctoral doivent être idéalement multimodales $7,10,14,15$. 


\section{Concepts et Innovations}

En conclusion, les séances d'ARE peuvent être aisément appliquées à la formation des résidents en anesthésieréanimation. Les intérêts pédagogiques de la méthode sont favorablement perçus par les apprenants et les tuteurs. Ces résultats sont suffisamment convaincants pour nous encourager à inclure les séances d'ARE dans notre curriculum postdoctoral. Il serait intéressant de mesurer l'impact de cette démarche pédagogique sur la performance des résidents dans l'identification et la résolution des problèmes éthiques par une approche collaborative et dialogique, au cours de leur pratique professionnelle. La place des séances d'ARE par rapport aux autres méthodes d'enseignement de l'éthique reste à déterminer.

\section{Contributions}

Alexandre Yazigi, Samia Madi-Jebara et Patricia Yazbeck ont participé solidairement à la conception et la réalisation de ce travail. Alexandre Yazigi a rédigé les différentes versions du manuscrit.

\section{Références}

1. Frank JR, Jabbour M, Tugwell P. Skills for the new millennium : report of the societal needs working group, CanMEDS 2000 project. Ann $R$ Coll Physicians Surg Can 1996;29:206-16.

2. Swick HM. Toward a normative definition of medical professionalism. Acad Med 2000;75:612-16.

3. Marcoux H, Patenaude J. L'éthique et la formation médicale: Où en sommes-nous? Où allons-nous? Pédagogie Médicale 2000;1:23-30.

4. Patenaude J, Lambert C, Dionne $M$, Marcoux $H$, Jeliu G, Brazeau-Lamontagne L. L'éthique comme compétence clinique: Modélisation d'une approche dialogique. Pédagogie Médicale 2001;2:71-9.

5. Barrier JH, Brazeau-Lamontagne L, Colin $R$, Quinton A, LLorca G Ehua FS et le conseil pédagogique de la CIDMEF. La formation au professionnalisme des futurs médecins. Recommandations $d u$ conseil pédagogique de la CIDMEF. Pédagogie Médicale 2004;5:75-81.

6. Chamberlain JE, Nisker JA. Residents' attitudes to training in ethics in Canadian obstetrics and gynecology programs. Obstet Gynecol 1995;85:783-6.
7. Angelos P, DaRosa DA, Derossis AM, Kim B. Medical ethics curriculum for surgical residents : Results of a pilot project. Surgery 1999;126:701-7.

8. Tallett SE, Belik J, Hilliard RI, Kenny NP, Rowell M, Lynch A. Ethics teaching and learning in pediatric training : development of a curriculum. Ann $R$ Coll Physicians Surg Can 1997;30:103-7.

9. Klein EJ, Jackson JC, Kratz L, Marcuse EK, McPhillips HA, Shugerman RP, et al. Teaching professionalism to residents. Acad Med 2003;78:26-34.

10. Edelstein SB, Stevenson JM, Broad K. Teaching professionalism during anesthesiology training. J Clin Anesth 2005;17:392-8.

11. Brazeau-Lamontagne L. L'évaluation de l'éthique médicale des résidents. Pédagogie Médicale 2002;3:152-8.

12. Brazeau-Lamontagne L. L'enseignement de l'éthique médicale - Réponse. Pédagogie Médicale 2002;3:200-1.

13. Syllabus on ethics 1999. American Society of Anesthesiologists; Committee of ethics. [On-line]. Disponible sur: http://www.asahq.org/publicationsAndServ ices/EthicsSyllabus.pdf 


\section{Les séances d'apprentissage au raisonnement éthique ...}

14. Arnold RM, Forrow L, Wartman SA, Teno J. Teaching clinical medical ethics : a model programme for primary care residency. J Med Ethics 1988;14:91-6.

15. Brownell AK, Coté L. Senior residents' views on the meaning of professionalism and how they learn about it. Acad Med 2001;76:734-7.

16. Tysinger JW, Klonis LK, Sadler JZ, Wagner JM. Teaching ethics using small-group, problem-based learning. J Med Ethics 1997;23:315-8.

17. Sheu SJ, Huang SH, Tang FI, Huang SL. Ethical decision making on truth telling in terminal cancer : medical students' choices between patient autonomy and family paternalism. Med Educ 2006;40:590-8.
18. Self DJ, Olivarez M, Baldwin DC Jr. The amount of small-group case-study discussion needed to improve moral reasoning skills of medical students. Acad Med 1998;73:521-3.

19. McAlpine H, Kristjanson L, Poroch D. Development and testing of the ethical reasoning tool (ERT) : an instrument to measure the ethical reasoning of nurses. J Adv Nurs 1997;25:1151-61.

20. SelfDJ, Baldwin DC Jr, Wolinsky FD. Evaluation of teaching medical ethics by an assessment of moral reasoning. Med Educ 1992;26:178-84.

Manuscrit reçu le 28 décembre 2006 ; commentaires éditoriaux formulés aux auteurs le 16 mai 2007 ; accepté pour publication le 6 juin 2007. 